\title{
Collaborative Data Delivery for Smart City-oriented Mobile Crowdsensing Systems
}

\author{
Piergiorgio Vitello ${ }^{\sharp}$, Andrea Capponi ${ }^{\dagger}$, Claudio Fiandrino ${ }^{\star}$, Paolo Giaccone ${ }^{\sharp}$, \\ Dzmitry Kliazovich ${ }^{\star}$ Ulrich Sorger ${ }^{\dagger}$, Pascal Bouvry ${ }^{\dagger}$ \\ \# Dip. di Elettronica e Telecomunicazioni, Politecnico di Torino, Italy, \\ ${ }^{\dagger}$ FSTC-CSC, University of Luxembourg, Luxembourg, * IMDEA Networks Institute, Madrid, Spain, ` ExaMotive, Luxembourg \\ E-mails: ${ }^{\sharp}$ piergiorgio.vitello@ studenti.polito.it, paolo.giaccone@ polito.it, ${ }^{\dagger}\{$ firstname.lastname $\} @$ uni.lu, \\ *claudio.fiandrino@imdea.org, kliazovich@ieee.org
}

\begin{abstract}
The huge increase of population living in cities calls for a sustainable urban development. Mobile crowdsensing (MCS) leverages participation of active citizens to improve performance of existing sensing infrastructures. In typical MCS systems, sensing tasks are allocated and reported on individual-basis. In this paper, we investigate on collaboration among users for data delivery as it brings a number of benefits for both users and sensing campaign organizers and leads to better coordination and use of resources. By taking advantage from proximity, users can employ device-to-device (D2D) communications like Wi-Fi Direct that are more energy efficient than 3G/4G technology. In such scenario, once a group is set, one of its member is elected to be the owner and perform data forwarding to the collector The efficiency of forming groups and electing suitable owners defines the efficiency of the whole collaborative-based system. This paper proposes three policies optimized for MCS that are compliant with current Android implementation of Wi-Fi Direct The evaluation results, obtained using CrowdSenSim simulator, demonstrate that collaborative-based approaches outperform significantly individual-based approaches.
\end{abstract}

\section{INTRODUCTION}

Smart cities aim to improve citizens' quality of life through an efficient use of ICT resources. Global population living in urban environments has significantly increased in the last century, reaching the $50 \%$ of the worldwide population and it is projected to grow further [1]. Urbanization calls for a rational development, which requires environmental monitoring and optimized use of resources. The Internet of Things (IoT) becomes a key for building effective sensing infrastructures [2]. Involving citizens ${ }^{1}$ in the sensing loop through mobile crowdsensing (MCS) is a win-win strategy [3]

With MCS, users contribute data generated by the rich set of sensors embedded in their mobile devices, such as smartphones, tablets and smart watches. Such data is delivered to a collector in the cloud for analytics. Intelligence and mobility of citizens provide a better context awareness and spatial coverage in respect to traditional sensor networks. Sensing is commonly employed in applications such as noise monitoring, health care and traffic management [4]. To illustrate with a few examples, HazeWatch [5] relies on citizen participation to monitor air pollution. Creekwatch [6] is an application for smartphones developed by the IBM Almaden research center for monitoring the conditions of watershed through crowdsensed data.

\footnotetext{
${ }^{1}$ In the remainder of the paper, we use the terms citizen, participant and user interchangeably.
}

In typical MCS systems, the allocation of sensing and reporting tasks is performed on individual-basis [7], [8]. However, collaboration among users could lead to a number of benefits for both users and sensing campaign organizers. For instance, users can take advantage from the proximity and use device-to-device (D2D) communications like Wi-Fi Direct that are more energy efficient than 3G/4G technology, which in turn will foster user participation [9], [10].

In this paper, we investigate how to make effective groupbased data delivery in MCS systems. MCS users are assumed to communicate using Wi-Fi Direct inside groups. Each group elects a group owner (GO) who is in charge to proxy and aggregate information from the group members delivering it to central collector. The election of the most suitable GOs defines the efficiency of the whole data delivery process. For example, a GO leaving the group too soon would preclude all members from reporting the collected data. We propose and compare performance of three different policies for group forming and subsequent GO election. In the first one, the sensing area is divided into a regular grid and users within a cell belong to a unique group. The second approach considers Point of Interests (PoIs), spatial locations where there is an interest to perform sensing and all users in the proximity of the PoI are associated to a group. Finally, the third policy simultaneously takes into account users' positions and mobility patterns and groups people that preferentially remain close to maximize group stability. The policies have been implemented in CrowdSenSim [11], which is the simulator employed for performance evaluation in realistic urban environments and has already been used for different smart city applications, such as smart lighting [12]. Collaborative-based data delivery improves significantly the energy costs the users would sustain performing individual-based reporting for group members that do not forward information to the collector.

\section{BACKGROUND}

Collaborative sensing bases its roots in forming groups among users in proximity. For example, in MCS all participants located within spatial and temporal proximity can be entitled to perform a task [13]. In ad-hoc networks, for data forwarding, routing techniques rely on position-based techniques to select the next-hop [14]. Peer-based communication technologies such as D2D are an excellent solution. D2D enables direct 
communication among mobile devices, thus allowing to better exploit radio resources. Specifically, D2D reduces interference, makes a better use of power allocation for transmission and thus limiting devices energy consumption [15]. Several technologies can be employed to enable D2D communications. LTE Direct and Wi-Fi Direct respectively foster D2D proximity discovery [16] and enables opportunistic networks [17] for local data dissemination. LTE Direct requires line-of-sight between devices as pre-requisite, while Wi-Fi Direct does not. In addition, the LTE interface is more power consuming than Wi-Fi [18]. Hence in this work we adopt the latter technology. The employed policy for group forming and GO election is crucial in collaborative MCS systems. User-performance can be boosted through protocols that manage the group size and the transmission power of each smartphone [19]. Optimizing the GO selection improves the overall network throughput [20]. In the context of MCS, mis-estimation of connectivity availability is detrimental for profit as users can lose rewards for not having successfully reported data. Exploiting collaboration among users in proximity and performing GO election efficiently alleviate this issue [21]. Unlike previous works, our aim is to propose energy-efficient collaborative solutions to form groups and to elect the GO, who coordinates sensing and reporting through D2D communications. Wi-Fi Direct supports multigroup communications as well. In [22], the authors present an Android application for unrooted devices able to form connected groups based on a generic metric for the election of the GO, overcoming the limitation of the legacy GO election. In our work, we consider a scenario in which all the devices belong to some groups, but multi-group communications are not exploited.

\section{Group-Based Collaborative SENSING}

The collaboration among users is beneficial to MCS systems in the following ways. First, it promotes a better energy management. Only a limited set of users, i.e., the GOs, are entitled to perform data delivery to the collector. The other users deliver the collected data to the GOs that are in their vicinity, hence limiting the energy consumption. Second, groupbased tasks allocation entitles MCS campaign organizer to fully benefit from the advantages of coordinated recruitment and to better control the spatio-temporal collection process, budget usage and quality of collected data.

In this paper, we consider Wi-Fi Direct technology. A Wi-Fi Direct Group is composed of a GO and zero or more group members (GM). A device can dynamically assume the role of GM or GO. According to [23], Wi-Fi Direct allows to form groups in standard, autonomous and persistent modes. In our paper, we exploit the autonomous group formation for different reasons. First, it does not require a negotiation phase as the standard mode, being faster and simpler with a device that announces itself as GO and starts a Wi-Fi Direct group sending beacons. Second, Wi-Fi Direct does not permit the standard and persistent group formation procedures among more than two devices. In our model, for each group the elected GO delivers data to the central collector through LTE interfaces. Only the

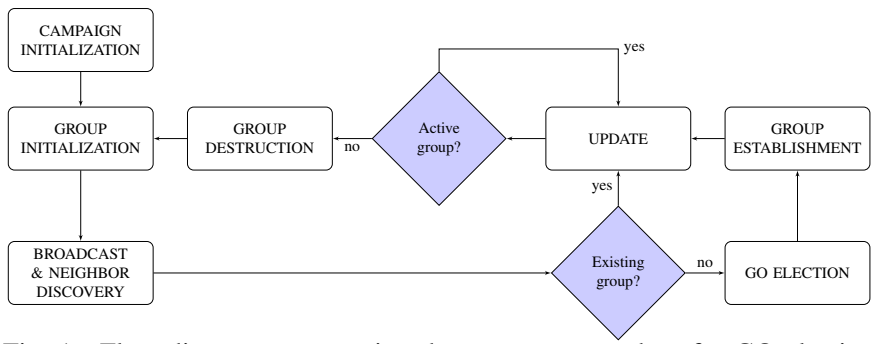

Fig. 1. Flow diagram representing the per-user procedure for GO election and group formation

participants that have previously subscribed the MCS campaign can be part of a Wi-Fi Direct group. During the campaign initialization, each device receives information about task allocation and policy for data delivery (see Sec. IV). By default, Android requires the users to manually authenticate before joining any Wi-Fi Direct group, which can be impractical for crowdsensing purposes. Hence, to overcome this limitation, the proposed solutions resort to the methodology proposed in [24] that allows devices to connect to each other automatically without any user intervention. Unlike other implementations, this methodology does not require to "root" the device.

The GO election is performed in distributed fashion by relying on the mechanisms introduced in [25] and parametrized to fit MCS purposes. Specifically, the rules for GO election, explained in detail in Sec. IV, incorporate additional parameters than the typical Wi-Fi Direct procedure that each policy exploits differently. Fig. 1 describes all the steps for group formation and GO election that are explained in detail in the following Section.

\section{The Proposed Policies for Group Formation AND GO ELECTION}

A MCS user $u$ is characterized by $I D_{u}, P_{u}, \alpha_{u}, V_{u}, B_{u}$, $R S S I_{u}, G R_{u}, G I_{u}, M_{u}, O_{u} . I D_{u}$ is the user identifier. $P_{u}$ is the position of the device (latitude and longitude). $\alpha_{u}$ is the azimuth of the user walking direction, given by the line connecting the position at the current timeslot $P_{u}^{t}$ and the previous one $P_{u}^{t-1}$. $V_{u}$ is the current user speed, $B_{u}$ is the remaining level of device battery and $R S S I_{u}$ is the received signal strength indicator. The parameters that also depend on the policy are the ID of the group $G R_{u}$ and the GO availability index $G I_{u}$, which defines the "fitness" of each user to become a GO. The intuition for the GO election is as follows. A user with higher battery level, RSSI and long-lasting remaining permanence in the group is a better GO candidate than another one with low battery, RSSI and about to leave the group. $M_{u}$ and $O_{u}$ are two flags that indicate if the device is a GM and a GO, respectively. During the phase of update and broadcast (see Fig. 1), these parameters advertise new users whether to join an active group or to start a GO election. The diagram describes the procedure when users join the group, while departures can occur in any stage.

We propose three policies for group formation and $\mathrm{GO}$ election. The first policy, Static Grouping (SG), is based on a regular grid that covers the whole area of interest and in each cell it exists at least one group (see Fig. 2(a)). The second one is the Point of interest Grouping (PG). This policy groups 


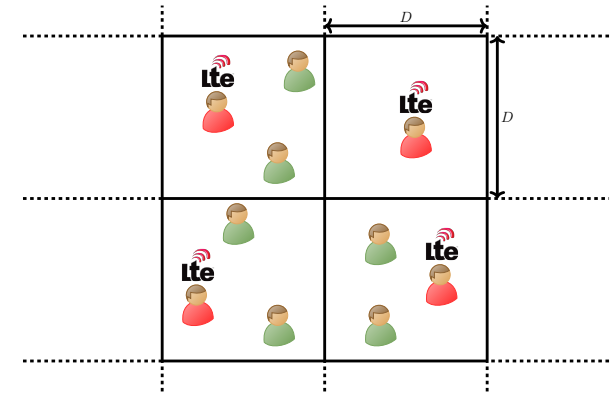

(a) Static Grouping (SG)

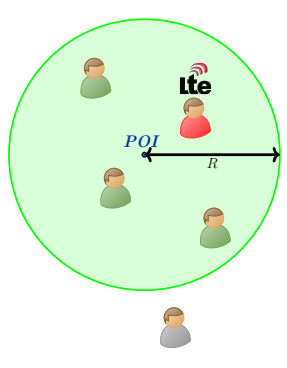

(b) Point of interest Grouping (PG)

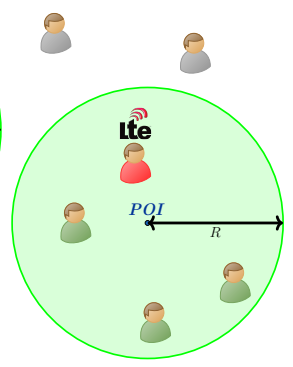

Fig. 2. Group formation for considered policies

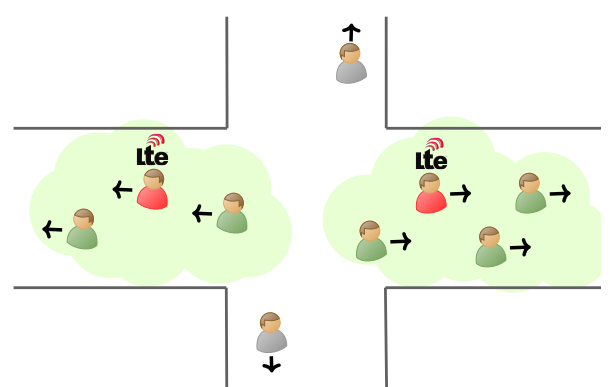

(c) Dynamic Grouping (DG)

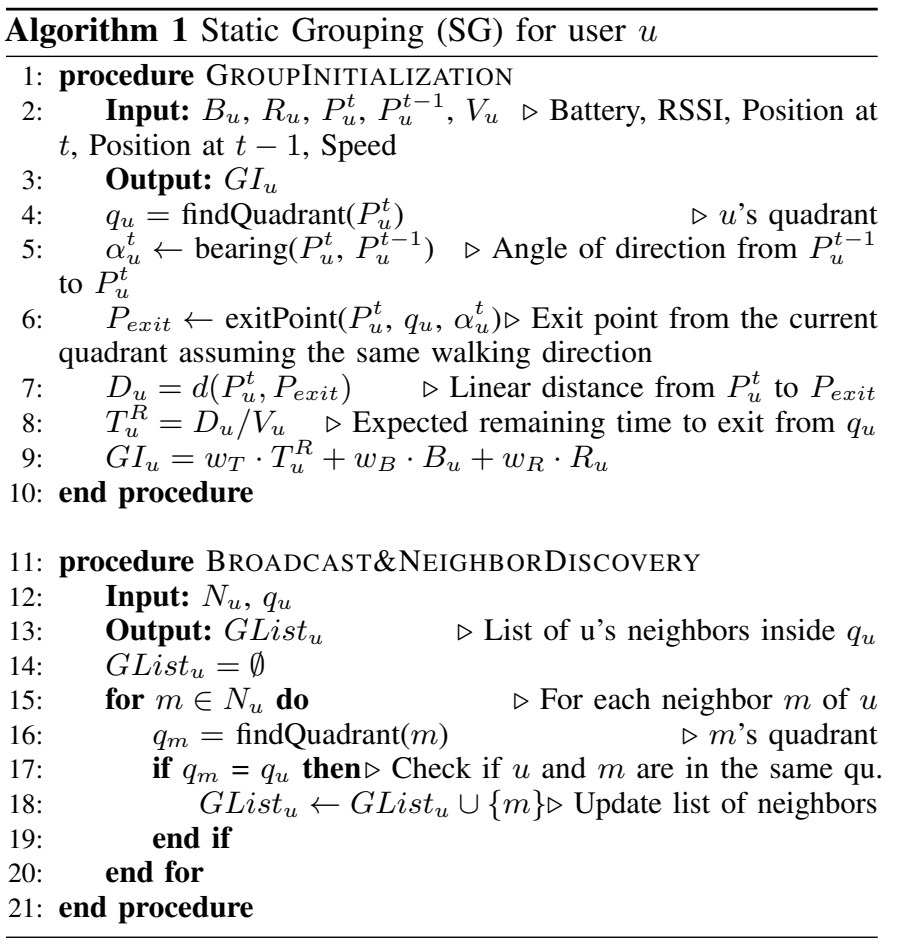

member on the basis of their distance from a given location, the PoI (see Fig. 2(b)). The last one is the Dynamic Grouping (DG), which forms groups remaining close for a certain time window (see Fig. 2(c)).

Static grouping (SG). The SG policy splits the region of interest into a regular grid. A group $G R_{u}$ is composed of all the users within the same cell. The approach is particularly useful for applications where full coverage of an area is required like in environment monitoring. Algorithm 1 presents the details of the Wi-Fi Direct SG-enabled procedure.

During the group initialization phase (see Fig. 1), each device determines its cell position. During broadcast and neighbor discovery, a mobile device exchanges with nearby devices in the same cell the list of parameters. $G I_{u}$ is computed as follows:

$$
G I_{u}=w_{T} \cdot T_{u}^{R}+w_{B} \cdot B_{u}+w_{R} \cdot R S S I_{u},
$$

where $T_{u}^{R}$ is the expected remaining time of the user $u$ in the current cell, computed as $T_{u}^{R}=D_{u} / V_{u}$, where $D_{u}$ is the distance between the current position of the user and the closed cell boundary location. To calculate $D_{u}$, it is assumed that the user will continue walking along the same direction given by $\alpha_{u}$. Finally, $w_{T}, w_{B}$ and $w_{R}$ are the weights that define the impact of corresponding components and are set by the MCS organizers to fulfill specific objectives of the campaign. When a new user enters a cell and a group is already formed, the device generates a new Glist and connects to the pre-existing group without electing a new GO. When the current GO leaves the cell, the current group is destroyed and the entire procedure of group formation and GO election starts over.

Point of interest grouping (PG). People tend to cluster in certain areas like railway stations, restaurants, or shopping centers, which can be defined as Points of Interest (PoI) and are often associated to category information in Location-Based Social Networks (LBSNs) [26]. Time plays an important role in PoIs because users tend to visit and remain in different places at different moments in a day (e.g., visiting a restaurant at lunchtime, or a pub at night) [27]. To model user behavior, a popular technique consists of analyzing check-in data obtained from LBSNs [28]. Hence, the PG policy builds on the sociability property and achieves longer group stability than SG at the price of lower area coverage. A group $G R_{u}$ is composed of users located at a distance lower than a certain radius $R_{P}$ from the location of a PoI. During the initialization phase, the devices set the parameters and receive all information needed for the campaign, such as the coordinates of PoIs. Once a device locates itself in an area corresponding to a PoI, it starts the broadcast and neighbor discovery phase to create the GList and form a group. $G I_{u}$ is now computed as follows:

$$
G I_{u}=w_{T} \cdot T_{u}^{S}+w_{B} \cdot B_{u}+w_{R} \cdot R S S I_{u},
$$

where $T_{u}^{S}$ is the expected dwell time within the PoI area, and $w_{T}, w_{B}$ and $w_{R}$ are the component weights. Differently from the SG policy, where a user stays for a certain time inside a cell depending on the movement, PG policy takes into account users stopping for a certain amount of time in a PoI to benefit from a service. Consequently, $T_{u}^{S}$ follows a distribution that depends on the typology of a PoI. When a new user arrives in a PoI and a group is already formed, the device creates a GList and connects to the pre-existing group without electing a new GO. A new GO is elected when the previous one leaves the PoI.

Dynamic grouping (DG). Users tend to group naturally while walking in streets [29]. DG takes advantage of this feature by creating Wi-Fi Direct groups among users that walk in similar 
direction. As in DG there is no notion of cell nor PoI that define the conditions for group destruction, the groups formed with DG are expected to last longer. Specifically, the DG policy creates a group when the users remain close one with each other for at least (KT) time period. The keeping time (KT) is defined as the expected time duration of two devices remaining under a certain radius 300 and is calculated based on the distance between users, their current velocity and walking azimuth. After the group initialization phase, during the broadcast and neighbor discovery phase each user creates a GList, computing for each GM the KT. If the value of KT is lower than the one associated to the threshold $\delta$, the corresponding node inside the GList is deleted. The value of $\delta$ varies according to the stability required from the MCS campaign. To elect the GO, each device computes its sociability $S_{u}$, which is defined as the number of neighbor devices satisfying KT. $S$ is the number of nodes inside the GList. GI is now computed as follows:

$$
G I_{u}=w_{S} \cdot S_{u}+w_{B} \cdot B_{u}+w_{r} \cdot R S S I_{u},
$$

where $w_{S}, w_{B}$ and $w_{R}$ are the component weights. During the update phase, a device performs the broadcast and neighbor discovery phase and there are three possibilities after the creation of a GList. First, the GList contains a GO and the device joins the existing group. Second, when GList contains other devices that are neither GMs nor GO and there are no existing group, the device starts from the beginning group formation and GO election. Finally, if there are GMs but no $\mathrm{GO}$, it means that GMs belong to a group in which the GO is unreachable. In this case the device deletes these GMs from the GList and performs a new group formation and owner election including only devices that do not belong to any group. When a GM loses contact with its GO, it starts again the group formation procedure. A group is destroyed only when no GMs are longer connected with a GO.

\section{A. Simulation set-up}

\section{Performance Evaluation}

For performance evaluation of the collaborative data-delivery policies, we use CrowdSenSim [11] to simulate the pedestrian movements in realistic urban environments. The users' trajectories are generated before the runtime simulation, as described in our former work [30]. For the experiments, we select Luxembourg city center, which covers an area of $51.47 \mathrm{~km}^{2}$ with a population of 116328 as of the end of 2017. Unless otherwise stated, we simulate a number of participants equal to 50000 . The walking period $T_{u}^{W}$ and the average speed $V_{u}$ of each participant are uniformly distributed in the range $[20,40]$ minutes and $[1,1.5] \mathrm{m} / \mathrm{s}$ respectively. Each user has only one device contributing data, with a battery level set to be uniformly distributed between $80-90 \%$ at the beginning of simulation. Users can exploit different models of smartphones and this choice defines the total battery capacity. The values are taken from a list of popular devices on market, including Huawei P8 Lite (2200 mAh), LG G5 (2800 mAh) and Samsung Galaxy J7 (3300 mAh).

Table I shows the simulation parameters exploited for sensing and communication equipment. Data contribution is implemented exploiting heterogeneous set of sensors typically embedded in popular smart devices, such as the MPU-6500 3axis linear accelerometer from InvenSense, the TMD4903 proximity sensor from AMS and the SKG13BL GPS module from SKYLAB. Table I(a) shows the sensing equipment exploited for the simulations and their corresponding parameters. To compute the power consumption of communication equipment, we refer to [31]. We consider the Wi-Fi Direct power consumption for uplink and downlink communications for local group data exchange. In addition, GO members report aggregated data to the cloud collector with LTE, hence only uplink LTE power consumption model is considered. Assuming uplink throughput is $T_{u}$ and downlink throughput is $T_{d}$, the instant Wi-Fi Direct power level for uplink is $P_{u}^{W}=\alpha_{u}^{W} \cdot T_{u}^{W}+\beta^{W}$ and for downlink $P_{d}^{W}=\alpha_{d}^{W} \cdot T_{d}^{W}+\beta^{W}$. The LTE power level for uplink is $P_{u}^{L}=\alpha_{u}^{L} \cdot T_{u}^{L}+\beta^{L}$. The best fitting parameters are listed in Table I(b), while throughput $T$ depend on curves that are taken from [31] for LTE communications and from [32] for Wi-Fi Direct.

The proposed policies are implemented as follows (see Table I(c) for a summary of the parameter details). The size of the groups changes over time according to the mobility and the proposed policies. The distance between members of the same group is at maximum the one allowed by Wi-Fi Direct standard. The area of each cell $A$ for the SG policy is of $500 \mathrm{~m}^{2}$, which is approximately the size of a microcell according to AT\&T standard. The PG policy exploits as PoIs the coordinates of 37 bus stops of the city center of Luxembourg, with a radius $R_{P}$ of 50 meters around the bus stop. The dwell time at a bus stop is uniformly distributed between $[1,15]$ minutes. DG exploits a distance $R_{D}$ of 50 meters around the $\mathrm{GO}$ and the threshold $\delta$ for the keeping time is set to 60 seconds. For all the policies, the weights are set equal to $1 / 3$. The maximum number of users $N_{\max }$ per each group is set to 10 .

\section{B. Simulation results}

For performance evaluation, we first compare energy consumption of individual and collaborative approaches. Then, we focus on specific characteristics of each policy, such as group duration, average number of members and role of users.

Fig. 3 shows the distribution of energy consumption with Individual reporting (Ind) and collaborative (SG, PG and DG) data delivery. Note that collaborative-based data forwarding policies outperform individual reporting as $75 \%$ of the users achieve lower power utilization. Such users, as better described in next results, are GMs. GO, instead, produces an extra-effort which leads to an increase of energy consumption with respect to Ind. Among the policies, PG is the one limiting the most the energy-consumption of GOs and achieves comparatively better performance than SG and DG. In PG, the number of users in the system that is not walking in close proximity of a PoI limits the chances for successful group creation.

Fig. 4 details the roles that users can assume, i.e., GO, GM or no-group. The latter category indicates users that are not in a group and do not contribute data. The reason for this category is twofold. First, users trying to join an already full group 
TABLE I

PARAMETERS OF SENSOR AND COMMUNICATION EQUIPMENT USED FOR PERFORMANCE EVALUATION

\begin{tabular}{llrl}
\hline SENSOR & PARAmETER & VAlue & Unit \\
\hline Accelerometer & Sample rate & 4 & $\mathrm{kHz}$ \\
& Sample size & 6 & $\mathrm{bytes}$ \\
& Current & 450 & $\mu \mathrm{A}$ \\
\hline \multirow{2}{*}{ Proximity } & Sample rate & 8 & $\mathrm{MHz}$ \\
& Sample size & 2 & $\mathrm{bytes}$ \\
& Current & 150 & $\mu \mathrm{A}$ \\
\hline \multirow{2}{*}{ GPS } & Update period & 10 & $\mathrm{~s}$ \\
& Sample size & 24 & bytes \\
& Current & 23 & $\mathrm{~mA}$ \\
\hline
\end{tabular}

(a) Sensor Equipment

\begin{tabular}{|c|c|c|c|}
\hline \multirow{3}{*}{ 壵 } & SYMBOL & VAlue & UNIT \\
\hline & $\alpha_{u}^{L}$ & 438.39 & $\mathrm{~mW} / \mathrm{Mbps}$ \\
\hline & $\beta^{L}$ & 1288.04 & $\mathrm{~mW}$ \\
\hline \multirow{3}{*}{ 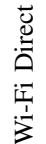 } & $\alpha_{u}^{W}$ & 283.17 & $\mathrm{~mW} / \mathrm{Mbps}$ \\
\hline & $\alpha_{d}^{W}$ & 137.01 & $\mathrm{~mW} / \mathrm{Mbps}$ \\
\hline & $\beta^{W}$ & 132.86 & $\mathrm{~mW}$ \\
\hline
\end{tabular}

(b) Communication Equipment

\begin{tabular}{lcl}
\hline SYMBOL & VALUE & UNIT \\
\hline$T_{u}^{W}$ & {$[20: 40]$} & $\mathrm{min}$ \\
$V_{u}$ & {$[1: 1.5]$} & $\mathrm{m} / \mathrm{s}$ \\
$w_{T}, w_{S}, w_{B}, w_{R}$ & $1 / 3$ & \\
$R_{P}, R_{D}$ & 50 & $\mathrm{~m}$ \\
$A$ & 500 & $\mathrm{~m}^{2}$ \\
$\delta$ & 60 & $\mathrm{~s}$ \\
$T_{u}^{S}$ & {$[1: 15]$} & $\mathrm{min}$ \\
$N_{\max }$ & 10 & \\
\hline
\end{tabular}

(c) Other parameters

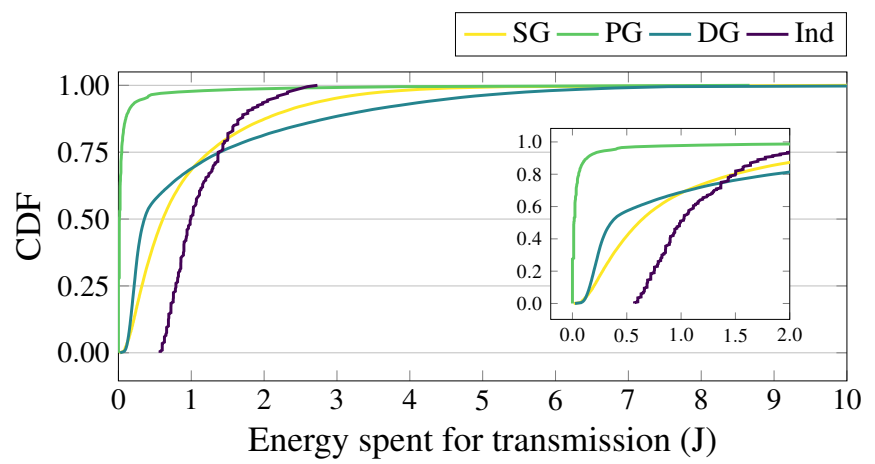

Fig. 3. CDF of energy consumption comparing all approaches (i.e., with already a maximum $N_{\max }$ of users) need to either wait and join another group, or wait that some of the existing members leave. Second, for PG and DG policies, users may be outside the range of PoI or of existing groups. The bars in the plot are computed as average over 100 simulation runs and the confidence interval is not graphically reported as too low. PG achieves the highest number of no-group users. Conversely, SG and DG form groups more consistently along time. In SG, the percentage of no-group users is higher than GO because in each grid is easy to reach the maximum number of participants. On the contrary, DG aims at maintaining groups as much as possible, hence the percentage of no-group users is lower. For all the policies, as expected, the number of GO users is lower than the number of GM users.

Fig. 5(a) shows the CDF of group duration. On one side, DG has the longest duration because groups remain active for longer periods (up to $30 \mathrm{~min}$ ). In SG, the group duration is less than 2 minutes for $90 \%$ of the cases because the GOs move and change often, causing group destruction and subsequent new GO election. The PG policy achieves results in between the former two, because the change of GO is less frequent than in SG, but more frequent than in DG. Fig. 5(b) shows the distribution of the variability of number of users in each group. The distribution is computed taking into account the number of group members in each group over a fixed time horizon. First, we observe that for all the policies the vast majority of groups are small, i.e., in 50\% of the cases SG-PG and DG exhibit groups of size smaller than 4 and 3 members respectively. Second, DG by design maintain groups longer and with higher number of members as follows people movements.

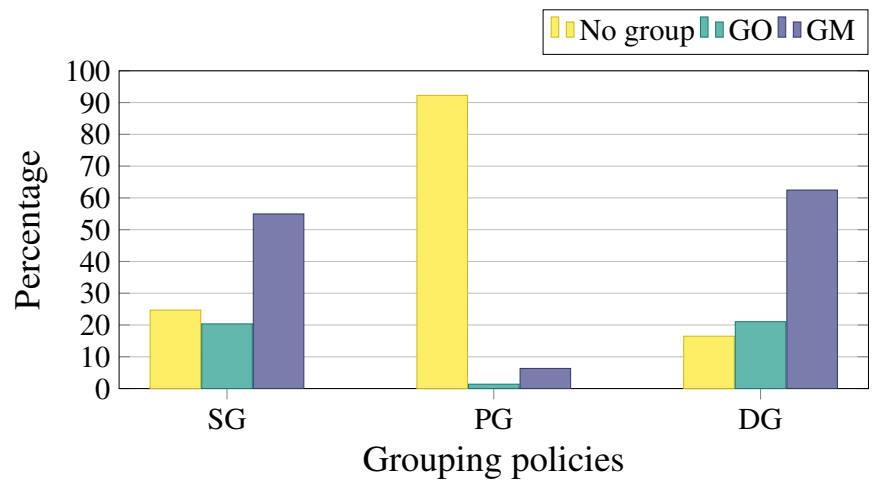

Fig. 4. Percentage of role of users for each grouping approach

Fig. 6 analyzes the energy consumption according to each user category. The per-minute energy consumption of GM users is the highest employing the SG policy, while the DG allows users to save energy as they remain within the same group longer. When users assume the role of GO, the distribution of energy consumption is similar for SG and DG. Comparing PG with the other policies, the median varies with difference up to $0.3 \mathrm{~J} / \mathrm{min}$. The reason is that, in $\mathrm{PG}$, the $\mathrm{GO}$ role changes more rapidly than with other policies. As expected, for no-group users the distribution of energy consumption remains identical for all the policies.

\section{CONCLUSION}

This paper presents an energy efficient group-based data delivery to accomplish tasks in MCS campaigns. To form groups and elect group owners, we propose three different policies suitable to different contexts and applications of a MCS campaign. The first policy constructs regular grid, where each cell represents a group, the second policy groups users around PoIs, while the third policy forms groups between pedestrians walking in the same direction along the streets. To conduct performance evaluation in a realistic urban scenario, Luxembourg City center, we resort to CrowdSenSim simulator. The results show that exploiting a collaborative data delivery approach brings significant energy savings for members of the group not responsible for data delivery to the collector. For future work, we plan to elaborate more on the proposed policies by implementing them and test in realistic urban settings.

\section{REFERENCES}

[1] N. B. Grimm, S. H. Faeth, N. E. Golubiewski et al., "Global change and the ecology of cities," in Science, vol. 319, no. 5864, 2008, pp. 756-760. 


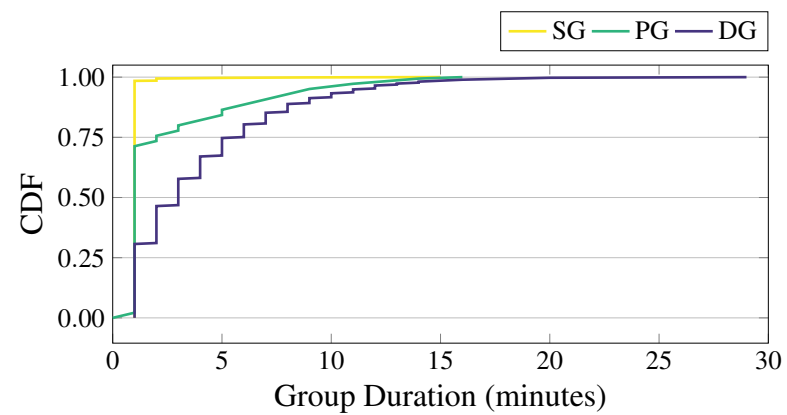

(a) Distribution of group duration

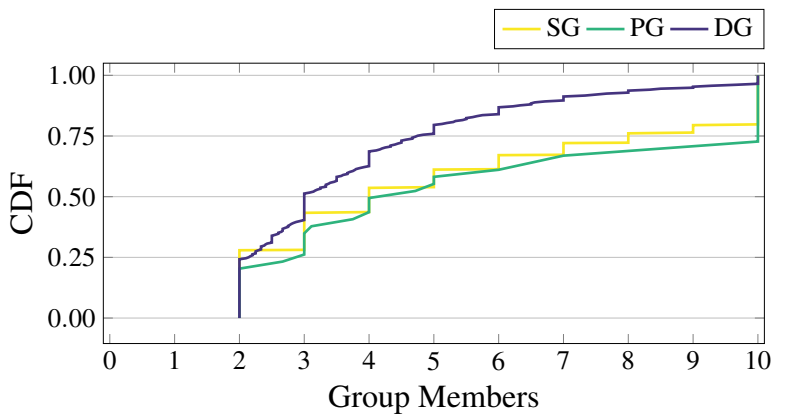

(b) Distribution of group size

Fig. 5. CDFs of group duration and group size for each grouping approach.

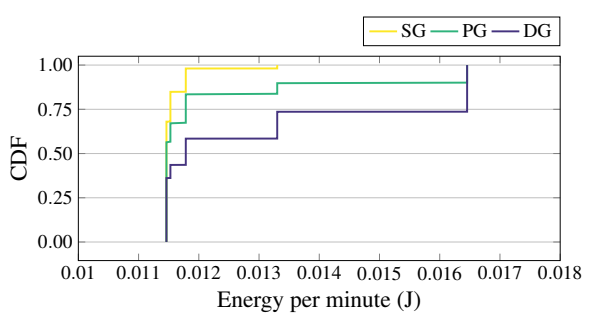

(a) Group member

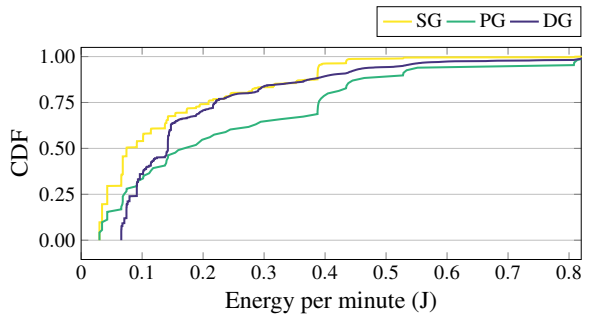

(b) Group owner

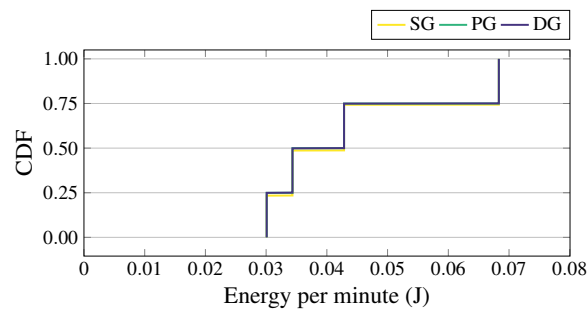

(c) No-group

Fig. 6. Energy consumption of different roles for each grouping approach.

[2] R. Ranjan, M. Wang, C. Perera et al., "City data fusion: Sensor data fusion in the internet of things," Int. J. Dist. Sys. Tech., vol. 7, no. 1, pp. 15-36, Jan 2016.

[3] G. Cardone, A. Cirri, A. Corradi et al., "Crowdsensing in urban areas for city-scale mass gathering management: Geofencing and activity recognition," IEEE Sensors Journal, vol. 14, no. 12, Dec 2014.

[4] W. Khan, Y. Xiang, M. Aalsalem et al., "Mobile phone sensing systems: A survey," IEEE Communications Surveys Tutorials, vol. 15, no. 1, pp. 402-427, First Quarter 2013.

[5] V. Sivaraman, J. Carrapetta, K. Hu et al., "HazeWatch: A participatory sensor system for monitoring air pollution in Sydney," in IEEE LCN, Oct 2013.

[6] S. Kim, C. Robson, T. Zimmerman et al., "Creek Watch: Pairing usefulness and usability for successful citizen science," in ACM SIGCHI, 2011, pp. 2125-2134.

[7] C. Fiandrino, B. Kantarci, F. Anjomshoa et al., "Sociability-driven user recruitment in mobile crowdsensing internet of things platforms," in IEEE GLOBECOM, Dec 2016, pp. 1-6.

[8] W. Gong, B. Zhang, and C. Li, "Task assignment in mobile crowdsensing: Present and future directions," IEEE Network, pp. 1-8, 2018.

[9] A. Capponi, C. Fiandrino, D. Kliazovich et al., "A cost-effective distributed framework for data collection in cloud-based mobile crowd sensing architectures," IEEE Trans. on Sustainable Computing, vol. 2 , no. 1, pp. 3-16, Jan 2017.

[10] M. Tomasoni, A. Capponi, C. Fiandrino et al., "Profiling energy efficiency of mobile crowdsensing data collection frameworks for smart city applications," in IEEE MobileCloud, Mar 2018, pp. 1-8.

[11] C. Fiandrino, A. Capponi, G. Cacciatore et al., "CrowdSenSim: a simulation platform for mobile crowdsensing in realistic urban environments," IEEE Access, vol. 5, pp. 3490-3503, Feb 2017.

[12] G. Cacciatore, C. Fiandrino, D. Kliazovich et al., "Cost analysis of smart lighting solutions for smart cities," in IEEE ICC, May 2017, pp. 1-6.

[13] L. Tran, H. To, L. Fan et al., "A real-time framework for task assignment in hyperlocal spatial crowdsourcing," ACM Trans. Intell. Syst. Technol., vol. 9, no. 3, pp. 1-26, Jan 2018.

[14] H. Füßler, J. Widmer, M. Käsemann et al., "Contention-based forwarding for mobile ad hoc networks," Ad Hoc Networks, vol. 1, no. 4, pp. 351 369, 2003.

[15] A. Asadi and V. Mancuso, "A survey on opportunistic scheduling in wireless communications," IEEE Communications Surveys Tutorials, vol. 15, no. 4, pp. 1671-1688, Fourth Quarter 2013.
[16] J. D. Benedetto, P. Bellavista, and L. Foschini, "Proximity discovery and data dissemination for mobile crowd sensing using LTE direct," Computer Networks, vol. 129, pp. 510 - 521, 2017.

[17] M. Conti, F. Delmastro, G. Minutiello et al., "Experimenting opportunistic networks with WiFi Direct," in IFIP Wireless Days (WD), Nov 2013.

[18] A. Asadi and V. Mancuso, "WiFi direct and LTE D2D in action," in IFIP Wireless Days, Nov 2013, pp. 1-8.

[19] M. Usman, M. Asghar, I. Ansari et al., "Towards energy efficient multihop D2D networks using WiFi Direct," in IEEE GLOBECOM, Dec 2017.

[20] K. Jahed, O. Farhat, G. Al-Jurdi et al., "Optimized group owner selection in WiFi Direct networks," in IEEE SoftCOM, Sep 2016, pp. 1-5.

[21] M. H. Cheung, F. Hou, and J. Huang, "Delay-sensitive mobile crowdsensing:algorithm design and economics," IEEE Trans. on Mobile Computing, 2018.

[22] C. E. Casetti, C. F. Chiasserini, Y. Duan et al., "Data connectivity and smart group formation in WiFi Direct multi-group networks," IEEE Trans. on Network and Service Management, Mar 2018.

[23] D. Camps-Mur, A. Garcia-Saavedra, and P. Serrano, "Device-to-device communications with WiFi-Direct: overview and experimentation," IEEE Wireless Communications, vol. 20, no. 3, pp. 96-104, Jun 2013.

[24] P. Wong, V. Varikota, D. Nguyen et al., "Automatic android-based wireless mesh networks," Informatica, vol. 38, 2014

[25] V. Arnaboldi, M. G. G. Campana, and F. Delmastro, "Context-aware configuration and management of WiFi direct groups for real opportunistic networks," in IEEE MASS, Oct 2017, pp. 266-274.

[26] M. Ye, P. Yin, W.-C. Lee et al., "Exploiting geographical influence for collaborative point-of-interest recommendation," in ACM SIGIR, 2011.

[27] Q. Yuan, G. Cong, Z. Ma et al., "Time-aware point-of-interest recommendation," in ACM SIGIR, 2013, pp. 363-372.

[28] A. Noulas, S. Scellato, C. Mascolo et al., "An empirical study of geographic user activity patterns in foursquare," in AAAI Conf. on Web and Social Media, 2011, pp. 1-4.

[29] A. Gorrini, S. Bandini, and M. Sarvi, "Group dynamics in pedestrian crowds: Estimating proxemic behavior," Journal of the Transportation Research Board, no. 2421, pp. 51-56, 2014.

[30] P. Vitello, A. Capponi, C. Fiandrino et al., "High-precision design of pedestrian mobility for smart city simulators," in IEEE ICC, May 2018.

[31] J. Huang, F. Qian, A. Gerber et al., "A close examination of performance and power characteristics of 4G LTE networks," in ACM MobiSys, 2012

[32] A. Pyattaev, K. Johnsson, S. Andreev et al., "3GPP LTE traffic offloading onto WiFi Direct," in IEEE WCNCW, 2013, pp. 135-140. 